\title{
Recruitment of Neuronal KCNQ2/3 Channels to Membrane Microdomains Depends on Palmitoylation of Alzheimer's Disease-Related Protein BACE1
}

\author{
Gucan Dai \\ Department of Physiology and Biophysics, University of Washington, Seattle \\ Correspondence: daig@uw.edu
}

Key words: KCNQ channels, BACE1, Alzheimer's disease, Palmitoylation, Lipid rafts

Running title: Recruiting KCNQ2/3 to Lipid Rafts by BACE1

Major and Minor classification: Biological Sciences; Physiology

\begin{abstract}
:
$\beta$-secretase 1 ( $\beta$-site amyloid precursor protein (APP)-cleaving enzyme 1, BACE1) plays a crucial role in the amyloidogenesis of Alzheimer's Disease (AD). BACE1 was also discovered to act like an auxiliary subunit to modulate neuronal KCNQ2/3 channels independent of its proteolytic function. BACE1 is palmitoylated at its carboxyl-terminal region, which brings BACE1 to ordered, cholesterol-rich membrane microdomains (rafts). However, the physiological consequences of this specific localization of BACE1 remain elusive. Using spectral Förster Resonance Energy Transfer (FRET), BACE1 and KCNQ2/3 channels were confirmed to form a signaling complex, a phenomenon that was relatively independent of the palmitoylation of BACE1. Nevertheless, palmitoylation of BACE1 was required for recruitment of KCNQ2/3 channels to lipid-raft domains. Two fluorescent probes designated L10 and S15, were used to label lipid raft and non-raft domains of the plasma membrane, respectively. Coexpressing BACE1 substantially elevated the FRET between L10 and KCNQ2/3 whereas the BACE1-4C/A quadruple mutation failed to produce this effect. In contrast, BACE1 had no significant effect on the FRET between S15 probes and KCNQ2/3 channels. A reduction of BACE1-dependent FRET between raft-targeting L10 probes and KCNQ2/3 channels by applying cholesterolextracting reagent methyl- $\beta$-cyclodextrin $(M \beta C D)$, raft-disrupting general anesthetics, or pharmacological inhibitors of palmitoylation all supported our hypothesis of the palmitoylationdependent and raft-specific localization of KCNQ2/3 channels. Furthermore, mutating the four carboxyl-terminal cysteines (4C/A) of BACE1 abolished the BACE1-dependent increase of FRET between KCNQ2/3 and a lipid raft-specific protein caveolin1. Collectively, we propose how the AD-related protein BACE1 underlies the localization of a neuronal potassium channel.
\end{abstract}

\section{SIGNIFICANCE STATEMENT:}

Alzheimer's disease (AD) has no effective cure. Animals with knock out of the key related enzyme BACE1 do not generate $\beta$-amyloids or develop $A D$, but rather exhibit altered behaviors and neuronal deficits. A new linkage between BACE1 and neuronal excitability is the interaction between BACE1 and KCNQ channels. These channels function as a brake for excessive neuronal excitability and are modulated by interacting with BACE1. We revealed a mechanism 
whereby BACE1 helps to recruit KCNQ channels to membrane microdomains called rafts. This localization of KCNQ channels is dependent on a specific type of lipidation of BACE1. This recruitment advances our understanding of $A D$ pathophysiology and could potentially inspire therapeutic interventions for the neuronal abnormalities associated with $A D$ treatments.

\section{INTRODUCTION}

This paper concerns a regulatory interaction of an Alzheimer's disease-related enzyme and a potassium ion channel. Alzheimer's disease (AD), the most prevalent form of dementia, is characterized by elevated amounts of amyloid plaque in the brain $(1,2)$. $\beta$-secretase 1 (BACE1) is one of the major proteases that cleave the amyloid precursor protein (APP), leading to the accumulation of amyloid $\beta(A \beta)$ fragments (3). A single mutation in APP that compromises the ability of BACE1 to cleave APP can reduce $A \beta$ generation and slow the progression of AD (4, 5). Therefore, inhibition of BACE1 has been proposed as a promising therapeutic strategy to treat AD (6). Indeed, mice with BACE1 gene knockout, BACE1(-/-), do not develop AD; however they do show changes in behavior tests, including spatial memory deficits, and sensorimotor impairments $(7,8)$. Indeed, BACE1(-/-) mice exhibit neuronal deficits including axonal organization defects in the hippocampus (9), myelination deficits (10) and altered synaptic function and neurotransmitter metabolism (11). In addition, BACE1(-/-) mice exhibit epileptic seizures, suggesting a possible direct role of BACE1 in regulating neuronal voltage-gated sodium and potassium channels $(12,13)$.

Candidate ion channels for regulation by BACE1 include the KCNQ family of voltage-gated potassium channels (Kv7) that conduct the neuronal M-current $(14,15)$. Two channel genes, KCNQ2 and 3, are widely expressed in the central nervous system (16); they form a heteromeric channel important for transducing sympathetic stimuli, dampening excessive neuronal excitability, reducing excitatory postsynaptic potentials and controlling neuronal firing $(17,18)$. KCNQ2/3 channels are abundantly present in the hippocampus and are critical for hippocampal excitability (19-22). In 2015, BACE1 was reported to interact with KCNQ2/3 channels directly to potentiate M-currents in hippocampus (15). Using patch-clamp electrophysiology, immunoprecipitation, and proximity-ligation assay, BACE1 was found to function as an auxiliary subunit of KCNQ2/3 channels, apparently independent of its proteolytic activity (15). Since KCNQ channel genes are well known for their involvement in several types of epilepsy $(23,24)$, this research seemingly provides a novel linkage between KCNQ2/3 channels and the epileptic phenotypes seen in BACE1(-/-) mice. Not surprisingly, it was also found that AD patients are more susceptible to develop epilepsy (25).

The enzymatic activity of BACE1 as an APP protease occurs in membrane lipid raft domains $(26,27)$. Lipid rafts are dynamic membrane microdomains containing sphingolipids, cholesterol, and certain proteins (28). Rafts are recognized as detergent-resistant membrane fractions, structurally more ordered than non-raft areas; they are signaling hubs orchestrating many cellular events (29). Lipid-raft localization of BACE1 biases the processing of APP in the amyloidogenic direction, generating $A \beta$ (27). When APPs are outside of raft domains, they are preferentially cleaved by the non-amyloidogenic $\alpha$-secretase $(30,31)$. Similar to other raftlocalizing proteins like tyrosine kinases, G protein $\alpha$-subunits, and purinergic P2X7 receptors, BACE1 uses palmitoylation, in this case, of four cysteines at its carboxyl-terminal end for specific membrane anchoring $(32,33)$. However, it is not well understood where the non- 
enzymatic activities of BACE1 localize, especially for regulating KCNQ channels. These channels do not have known palmitoylation sites, but are reported to interact with palmitoylated scaffold proteins, including A-kinase anchoring proteins (AKAP79 in human) and ankyrin-G (3437). Furthermore, previous research suggested a spatial overlap in the subcellular localization of BACE1 and KCNQ channels; KCNQ channels are thought to be localized in axons and axon initial segments (AIS) via ankyrin-G, whereas BACE1 is abundant in axons and implicated functionally in the myelination of axons $(10,34,38)$.

To understand the regulation of KCNQ2/3 channels by BACE1, we looked for localization of the BACE1-KCNQ complex in the lipid rafts of cultured cells and provided further evidence supporting the interaction of BACE and KCNQ2/3. We used Förster Resonance Energy Transfer (FRET) to demonstrate the proximity of membrane proteins and measured FRET, in a spectral manner, based on the sensitized emission of FRET acceptor after exciting the FRET donor. Using engineered fluorescent probes derived from short lipidated sequences of protein kinases Lck and Src to label lipid raft and non-raft domains respectively $(39,40)$, we showed that KCNQ channels are localized to lipid rafts. This specific localization of KCNQ channels is dependent on the palmitoylation of BACE1. We provide a strategy to determine the localization of proteins in specific lipid microdomains that could be broadly applied to answer similar questions of other membrane proteins.

\section{RESULTS}

This article reports the specific localization of neuronal KCNQ2/3 channels in membrane microdomains (lipid rafts), controlled by formation of a complex with BACE1.

\section{FRET between KCNQ2/3 and BACE1 consistent with their close assembly}

BACE1 interacts with KCNQ2/3 channels directly. Since some KCNQ channels can assemble with single-transmembrane auxiliary subunits e.g., KCNE1 for cardiac KCNQ1 channels (17); BACE1, which is also a single-transmembrane protein, has been considered by analogy as a novel auxiliary subunit for KCNQ2/3 channels (15). Atomic details of the interaction between BACE1 and KCNQ2/3 are largely unknown. Here, we further tested this interaction using FRET between a fluorescent noncanonical-amino acid L-Anap $(41,42)$ (Fig. S1) incorporated into the carboxyl-terminus of a mouse BACE1 and yellow fluorescent protein (YFP) fused to the carboxyl-terminus of both human KCNQ2 and KCNQ3 subunits all expressed in a cell line. KCNQ2 and KCNQ3 subunits form heteromeric channel complexes and were expressed together throughout this paper in the tsA201 cell line derived from human embryonic kidney cells.

BACE1 consists of an extracellular (amino-terminal) proteolytic domain, one helical transmembrane domain and a short intracellular (carboxyl-terminal) region (Fig. 1A). Four cysteines, one near the cytoplasmic end of the transmembrane domain(C474) and three within the intracellular region (C478, C482, C485) are S-palmitoylated, giving the lipid raft-localization of BACE1. Mutating these cysteines is reported to reduce the raft localization of BACE1 and amyloid burden but does not abolish the processing of APP by BACE1 $(33,43)$. The DXXLL motif in the carboxyl-terminus of BACE1 (Fig. 1A) is involved in the endocytosis and retrograde transport of BACE1 to the trans-Golgi network $(44,45)$. Mutating this motif increases the plasma 
membrane localization of BACE1 (45). In addition, the transmembrane and carboxyl-terminal of BACE1 are highly conserved (almost identical) among different species.

Considering the small size of the intracellular carboxyl-terminal part of BACE1, fusing a large GFP-derived fluorescent protein might perturb the natural properties of BACE1, so a fluorescent noncanonical amino acid L-Anap was incorporated at the carboxyl-terminal end of the BACE1. An amber stop-codon (TAG) suppression strategy (41) was used to incorporate L-Anap at the leucine 500 (L500) position within the DXXLL motif of BACE1 (asterisk, Fig S1). To express BACE1-L500Anap in tsA201 cells, cells were transiently cotransfected with the BACE1L500TAG cDNA and a plasmid pANAP encoding for the orthogonal amber suppressor tRNA/aminoacyl-tRNA synthetase (aaRS) pair for L-Anap and incubated overnight with culture medium supplemented with $20 \mu \mathrm{M}$ of the membrane-permeable methyl-ester form of L-Anap (46). When the translation machinery arrives at the TAG stop-codon site, translation continues by including L-Anap and, avoiding chain termination. This strategy has previously been shown to achieve satisfactory incorporation of L-Anap to exogenously-expressed recombinant membrane proteins $(47,48)$. In our experiments, cells grown without applying pANAP plasmid (tRNA/aaRS), but with BACE1-L500TAG cDNA transfected and L-Anap in media were used as negative controls for the FRET experiments.

The Anap emission spectrum overlaps well with the excitation spectrum of YFP, making them a practical FRET pair $(41,47)$. A high FRET would indicate close proximity of the two proteins. FRET between L-Anap and YFP was determined by calculations from the emission spectra of both fluorophores measured using a spectrometer attached to the microscope. This type of spectrum-based FRET includes corrections for the bleed-through and the cross-talk due to spectral properties of FRET donors and acceptors (see Methods). Cells that expressed BACE1L500TAG alone were used to generate a template L-Anap emission spectrum (cyan spectrum in Fig. 1B) to correct for bleed-through of the Anap emission in the wavelength range for YFP emission. In addition, cells that expressed only KCNQ2/3-YFP channels (no FRET donor) were used to determine a cross-talk correction for FRET: namely when excited by the Anap excitation light, they generated an emission spectrum whose amplitude was $6 \%$ of the YFP emission when directly excited by YFP excitation (Fig. 1C). The 6\% correction had to be subtracted from the FRET spectrum. Finally, to determine the FRET spectrum, the fluorescence spectrum was measured when both labeled BACE1 and KCNQ2/3-YFP channels were present (green spectrum), the cyan Anap template spectrum in Fig. 1B was then scaled and subtracted giving the resulting $F_{F R E T}$ spectrum (red). This noticeable amplitude of the red $F_{F R E T}$ spectrum in Fig. $1 \mathrm{~B}$ suggests that a population of Anap-labeled BACE1 molecules was in close proximity with KCNQ2/3-YFP channels.

The calculated apparent FRET efficiency varied with the ratio of expression of L-Anapincorporated BACE1 relative to KCNQ2/3-YFP. The apparent FRET efficiency would be linearly proportional to the absolute FRET efficiency occurring in the system and correlated positively with the ratio of Anap to YFP peak fluorescence intensities (Fig. 1B and D). Such a positive correlation is typical for ensemble FRET with variable fluorophore stoichiometries $(49,50)$. Note that the fluorescence ratio $F(A n a p) / F(Y F P)$ here in Fig 1D is not equal to the molar ratio of Anap versus YFP, but rather is also dependent on our optical apparatus and on the FRET efficiency, since FRET decreases Anap fluorescence intensity while increasing that of YFP. Essentially, there are different populations of fluorescent molecules regarding FRET: donors alone, acceptors alone, and donors paired with acceptors. Since detailed information about 
stoichiometry, contact site and affinity of the BACE1 and KCNQ2/3 interaction was lacking, fitting the relationship between the $F($ Anap) / F(YFP) ratio and the apparent FRET efficiency using published models with certain assumptions $(51,52)$ was not feasible. Therefore, a simpler, less model-dependent Langmuir isotherm was used for an empirical presentation of the data in Fig. 1D. Later in the paper, we also used this spectral FRET analysis for the cyan fluorescent protein (CFP) and YFP pairs (see Methods).

The apparent FRET efficiency between BACE1-L500Anap and KCNQ2/3-YFP did not change (no statistical difference) when the four palmitoylatable cysteines in BACE1 were mutated to alanines (4C/A) or when cells were incubated with the palmitoylation inhibitor 2-bromopalmitate (53) (0.1 mM 2-BP) (Fig. 1D and E). Negative-control cells lacking transfected pANAP plasmid showed minimal FRET even though visible Anap fluorescence was present due to nonspecific entry of L-Anap to cells. Furthermore, another voltage-gated potassium channel KCNH (a zebrafish ELK) (48), that was considered to be structurally similar to KCNQ but to have no reported interaction with BACE1, showed negligible FRET (Fig 1D and E). In addition, rather than comparing all the apparent FRET efficiency measurements for each condition by using averaged values (as in Fig. 1E), the FRET efficiencies at a fixed $F($ Anap) / F(YFP) ratio of 1 were also compared among different conditions; these efficiencies were: $0 \%$ in the absence of plasmid pANAP, 4.8\% for wild-type BACE1, 4.4\% for mutant BACE1-4C/A, 4.7\% after adding 2$\mathrm{BP}$ and $0.7 \%$ for BACE1/KCNH. These results, together with previously-published work (15), suggested that BACE1 and KCNQ2/3 channels form a protein complex that is relatively independent of the palmitoylation of BACE1.

\section{BACE1-dependent proximity between KCNQ2/3 and the raft-protein caveolin1}

If KCNQ2/3 forms a complex with BACE1, is it localized to lipid-rafts? We used the protein caveolin 1, a principal component of caveolae localized to lipid rafts $(50,54,55)$, to answer this question. Caveolin1 often dimerizes at its amino-terminal region and uses the palmitoylation of three cysteines at its carboxyl-terminus to target to lipid rafts $(50,55)$. A carboxyl-terminal CFPtagged caveolin 1 was coexpressed with KCNQ2/3-YFP channels in tsA cells, and their FRET was measured using spectral FRET. Without BACE1, the FRET between caveolin 1-CFP and KCNQ2/3-YFP was modest (Fig 2A, E and F). As before, the apparent FRET efficiency increased as the ratio CFP/YFP increased (Fig. 2E). The relationship between the apparent FRET efficiency and the CFP/YFP ratio was fitted empirically with the Langmuir isotherm (Fig. $2 \mathrm{E})$. Lacking additional evidence of direct complex formation, it is possible that caveolin1 and KCNQ2/3 might not interact directly like BACE1 with KCNQ2/3. Instead, they might FRET nonspecifically because of a sufficiently high local density $(49,50)$. However, with the coexpression of BACE1, there was a large increase in the apparent FRET intensities. In contrast, with coexpression of the BACE1-4C/A mutant construct, there was no increase in the CFP-YFP FRET, and the relationship between FRET and donor/acceptor intensity ratio superimposed on that seen without BACE1. Comparison of these conditions was best done at a fixed ratio of $F(C F P) / F(Y F P)$, so the representative FRET spectra chosen in Fig. 2 A-C had similar ratios of CFP/YFP intensity. At a fixed $F(C F P) / F(Y F P)$ ratio of 3 , the apparent efficiencies were: $4.8 \%$ in the absence of BACE1, $15.9 \%$ for wild-type BACE1, and $5.4 \%$ for mutant BACE1-4C/A. Together, these results suggested that, with the help of BACE1, overexpressed KCNQ2/3 channels were drawn to lipid-raft domains where they FRETed more efficiently with raft-specific caveolin1. Endogenous BACE1 is reported to be present in human embryonic kidney cells (based on the Human Protein Atlas, proteinatlas.org), but in our 
overexpression system, there must have been too little endogenous BACE1 or other scaffold proteins to occlude the actions of additional overexpressed BACE1.

\section{Using fluorescent probes that target lipid raft and non-raft domains}

To further study the lipid raft-localization of KCNQ2/3 channels, we turned to two fluorescent probes that can be targeted to liquid-ordered and -disordered domains at the plasma membrane (Fig. 3A). The L10 probe derives from the first 10 amino acids of the amino terminus of Lck kinase, which contains two palmitoylation sites for localizing to the ordered lipid-raft domains $(39,40)$. In contrast, the S15 probe derives from the first 15 amino acids from the amino terminus of Src kinase, which contains one myristoylation site, for localizing to the disordered non-raft domains $(39,40)$.

Validation experiments were first performed comparing the FRET between the Caveolin 1-CFP and L10-YFP pair to FRET between the Caveolin 1-YFP and S15-YFP FRET pair (Fig 3 B-D). As expected, FRET between L10 and Caveolin 1 was much higher than between $\mathrm{S} 15$ and Caveolin 1, reflecting a colocalization of Caveolin 1 with L10 in raft microdomains. Consistent with previous research, plotting the relationship between the apparent FRET efficiency versus the CFP/YFP ratio suggested a saturation curve, that might indicate a clustering of fluorescent molecules in a small area. Since lipid rafts are considered small ( $<250 \mathrm{~nm}$ in diameter), conventional fluorescence microscopy that is limited by the diffraction limit of light cannot visualize them directly. Our FRET method provides an alternative to measure the colocalization of proteins in small raft areas quantitatively (also see Myeong et al., PNAS, in press, 2021).

Next, L10 and S15 probes were used to test for possible colocalization with KCNQ2/3 channels and to learn how it might be regulated by BACE1. L10-CFP probes were coexpressed with KCNQ2/3-YFP channels without BACE1. The FRET between L10-CFP and KCNQ2/3-YFP was small (black curve, Fig $4 \mathrm{~A}$ and $\mathrm{C}$ ). Coexpression of BACE1 significantly elevated the FRET (red curve), whereas coexpression of the mutant BACE1-4C/A did not (blue curve). At a fixed F(CFP) / F(YFP) ratio of 4, the apparent efficiencies for the L10-CFP / KCNQ2/3-YFP pair were: $2.5 \%$ in the absence of BACE1, 13.4\% for wild-type BACE1, and 3.7\% for mutant BACE1-4C/A. However, using the same experimental settings, neither BACE1 nor the mutant BACE1-4C/A construct had an effect on the FRET between S15-CFP and KCNQ2/3-YFP (Fig 4 B and D). At a fixed $F(C F P) / F(Y F P)$ ratio of 4, the apparent efficiencies for the S15-CFP / KCNQ2/3-YFP pair were: $5.8 \%$ in the absence of BACE1, 5.8\% for wild-type BACE1, and $5.3 \%$ for mutant BACE1-4C/A. In addition, a higher apparent FRET efficiency for KCNQ2/3-YFP with S15-CFP than with L10-CFP without coexpression of BACE1 (replotted and highlighted in Fig S3B), suggests that KCNQ2/3-YFP channels by themselves may be localized more in the non-raft areas, consistent with their lack of known palmitoylation sites. This conclusion is predicated on the assumptions that endogenous BACE1 levels are low and that CFP fluorophores in L10 and S15 probes have comparable distances to the membrane and dipole orientations. To summarize, overexpressed KCNQ2/3 channels initially showed somewhat higher colocalization with S15 than with L10 (highlighted in Figure S3 B), however, coexpressing BACE1 significantly increased the localization of KCNQ2/3 channels to lipid rafts. With BACE1, KCNQ2/3 showed higher FRET with L10 than with S15 presumably due to a direct interaction between KCNQ and BACE1. Mutating the 4 cysteines in BACE1 to alanine eliminated this effect, corroborating a critical role of palmitoylation for the lipid raft-localization of both BACE1 and its partner KCNQ2/3. 
Several additional pharmacological manipulations were applied to cells that showed high FRET between L10-CFP and KCNQ2/3-YFP in the presence of wild-type BACE1 (Fig. 4 E and F). We applied cholesterol-extracting methyl- $\beta$-cyclodextrin (MBCD) in the medium, which could efficiently decrease plasma membrane cholesterol levels and reduce the relative area fraction of rafts (56). M $B C D$ application (5 mM for 5-10 mins) decreased the FRET between L10-CFP and KCNQ2/3-YFP considerably, suggesting that this FRET is dependent on the domains as well as on cholesterol that helps to maintain the rafts (Fig. $4 \mathrm{E}$ and F). Inhaled general anesthetics also disrupt lipid rafts at anesthetic concentrations (57). We found that one general anesthetic chloroform (1 mM) dramatically suppressed the FRET between L10-CFP and KCNQ2/3-YFP, similar to the effect of $M \beta C D$. In addition, 2-BP $(0.1 \mathrm{mM})$, an inhibitor of palmitoylation, abolished the FRET, consistent with a role of palmitoylation in the FRET signals we measured (Fig $4 \mathrm{E}$ and $\mathrm{F}$ ).

\section{Similar distribution of $\mathrm{PI}(4,5) \mathrm{P}_{2}$ in rafts versus non-rafts}

Lastly, we explored one possibility for the mechanism of the regulation of KCNQ2/3 channel function by BACE1, focusing on the membrane phospholipid phosphatidylinositol 4,5bisphosphate $\left(\mathrm{PI}(4,5) \mathrm{P}_{2}\right) . \mathrm{PI}(4,5) \mathrm{P}_{2}$ is a signaling lipid required for KCNQ2/3 channel activation (58-60). A YFP-tagged $\mathrm{PI}(4,5) \mathrm{P}_{2}$ sensor Tubby was used to label $\mathrm{PI}(4,5) \mathrm{P}_{2}$ in cells $(60)$. Resting FRET efficiencies between Tubby-YFP and either L10-CFP or S15-CFP were high and indistinguishable in amplitude (Figure S3) suggesting that $\mathrm{PI}(4,5) \mathrm{P}_{2}$ is equally present in lipid raft domains and non-raft domains of the plasma membrane, in agreement with Myeong et al. (2021). Future research will be required to test whether $\mathrm{PI}(4,5) \mathrm{P}_{2}$ is involved directly in the change of KCNQ2/3 function by forming the complex with BACE1 (15).

\section{DISCUSSION}

Using FRET, as a spectroscopic distance ruler, we found that KCNQ2/3 channels are in proximity to lipid-raft markers and to raft-specific proteins. We propose that neuronal KCNQ2/3 channels are localized to or close to the ordered membrane microdomain lipid rafts. This localization is achieved by the interaction between KCNQ2/3 and BACE1 and is dependent on the S-palmitoylation of 4 cysteines in the BACE1 carboxyl terminus. Without BACE1, KCNQ2/3 channels at the plasma membrane might be distributed more in the disordered non-raft areas at least in our heterologous expression system. Palmitoylation alters the ion channel localization and affects KCNQ channels indirectly via their auxiliary subunit BACE1 in this case. This type of ion channel regulation by S-palmitoylation is distinct from the direct lipidation and anchoring of ion-channel domains to lipid bilayers, thereby changing its gating; for instance, palmitoylation of the $\mathrm{P} 2 \mathrm{X}_{7}$ ATP receptor prevents its desensitization (61). However, this indirect regulation is analogous to the inhibition of the cardiac sodium/potassium pump by palmitoylation of the auxiliary phosphoprotein phospholemman (62).

Palmitoylation manifests an important way for localizing cell signaling ranging from cellular excitability to massive endocytosis $(32,63)$. For membrane proteins, S-palmitoylation is reversible and controlled by Asp-His-His-Cys acyltransferases (e.g., plasma membrane DHHC5), thought to be abundant in ordered lipid microdomains $(32,62)$. The palmitoylationdependence of KCNQ2/3 localization to rafts suggests that the channel function could also be indirectly affected by this acyltransferase. Specifically, it is reasonable to predict that KCNQ channel function could be modulated by cellular signals that are upstream of DHHC, like 
generation of reactive oxygen species (ROS) (64), opening of mitochondrial permeability transition pores (PTPs), coenzyme A (CoA) release, etc. $(63,65)$. Furthermore, in native neuronal environments, KCNQ2/3 channels are localized to axons by interacting with palmitoylated ankyrin $\mathrm{G}$, which itself could possibly recruit KCNQ2/3 channels to lipid rafts (34, 36). This ankyrin-G interaction could also facilitate the interaction of KCNQ2/3 with BACE1. The possible synergistic effect of the palmitoylations of KCNQ2/3-associated proteins including ankyrin G, BACE1 and AKAP79 remains elusive.

This research provides a new function for the raft-localization of BACE1, perhaps independent of the proteolytic or amyloidogenic features of BACE1. Previous research has shown that abolishing the raft-localization of BACE1 reduces the amyloid production and mitigates memory deficits in transgenic mouse models of Alzheimer's disease (43). Conversely, targeting BACE1 to lipid rafts by a glycosylphosphatidylinositol (GPI) anchor elevates the $\beta$-site processing of the amyloid precursor protein (26). Despite these findings, this role of the raft-localization of BACE1 for APP processing is still debated; other research suggested that the A $\beta$ production was still present even in the absence of the palmitoylation-dependent targeting of BACE1 to lipid rafts (33). Here, the raft-localization of BACE1 for the KCNQ2/3 channel function could play a role in reducing neuronal electrical excitability (22), modulating afterhyperpolarization potentials (AHP) $(66,67)$ and maintaining the proper propagation of nerve spikes $(20)$. It remains to be determined whether KCNQ2/3 channel activity affects the ability of BACE1 to cleave APP and to generate amyloid- $\beta$ peptides reciprocally.

Disruption of lipid rafts is proposed to underlie the mechanism for the medical effect of inhaled general anesthetics (57). Lipid-soluble general anesthetics change the lipid organization of sphingomyelin- and cholesterol-rich rafts and displace phospholipase D (PLD) from rafts to nonraft areas, which in turn generates phosphatidic acid (PA) that activates $K_{2 P}$ (TREK) ion channels (57). Here, applying chloroform was able to decrease the FRET between KCNQ2/3 with the raft-marker L10, indicating that general anesthetics similarly displace KCNQ2/3 to nonraft areas and could possibly alter the activity of the channels. Since we think $\mathrm{PI}(4,5) \mathrm{P}_{2}$ is present in both rafts and non-rafts, and relatively-uniformly distributed at the plasma membrane, other molecular components intrinsic to the lipid rafts might contribute more to the effect of BACE1 on KCNQ2/3. Furthermore, hydrolysis of $\mathrm{PI}(4,5) \mathrm{P}_{2}$ did not seem to affect the electrophysiological changes of KCNQ2/3 caused by BACE1 coexpression (15). Future research will be needed to further understand the detailed molecular mechanism underlying the regulation of KCNQ2/3 channels by BACE1. 


\section{MATERIALS AND METHODS}

\section{Cell Culture, Molecular Biology and Reagents}

The tsA-201 cells were cultured, maintained and transfected as previously reported (60). Point mutations were made using Quickchange II XL Site-Directed Mutagenesis kit (Agilent technologies, Santa Clara, CA). The sequences of the DNA constructs were confirmed by fluorescence-based DNA sequencing (Genewiz LLC, Seattle, WA). The mBACE1 construct was obtained from Addgene (Cambridge, MA). L-Anap (AsisChem, Waltham, MA) was made as a 10 $\mathrm{mM}$ stock, stored at $-20^{\circ} \mathrm{C}$ and diluted by 500 -fold into culture medium. The fluorescent L10 and S15 constructs were made in the lab of Dr. Byung-Chang Suh at Daegu Gyeongbuk Institute of Science and Technology (South Korea) (Myeong et a., PNAS, in press, 2021). The caveolin 1 construct was from Suzanne F. Scarlata (Worcester Polytechnic Institute, MA). The pANAP plasmid (Addgene, Cambridge, MA) contained the orthogonal tRNA/aminoacyl-tRNA synthetase specific to L-Anap. 2-bromopalmitate (2-BP) was delivered with fatty acid-free bovine serum albumin (Sigma-Aldrich, A6003) at a stock concentration $1 \mathrm{mM}$ albumin with 5 $\mathrm{mM} 2-\mathrm{BP}$, which was diluted by 50 -fold into the culture medium generating a final concentration of $0.1 \mathrm{mM} 2-\mathrm{BP}$.

\section{Quantification of FRET and Spectroscopic Analysis}

A Nikon Eclipse TE2000-E inverted microscope with a 60X, 1.2 NA water immersion objective was used for fluorescence measurements. Epifluorescence recording was performed with widefield excitation using a Lambda LS Xenon Arc lamp (Sutter Instruments). For spectral measurements, images were collected by a spectrograph (Model: $2150 \mathrm{i}, 300 \mathrm{~g} / \mathrm{mm}$ grating, blaze $=500 \mathrm{~nm}$; Acton research, Acton, MA) mounted between the output port of the microscope and an Evolve 512 EMCCD camera (Photometrics, Tucson, AZ) as previously reported (47). A filter cube containing a 376 (centered)/30 (width) nm excitation filter was used for L-Anap. CFP was excited with a filter cube containing a $425 / 40 \mathrm{~nm}$ excitation filter whereas YFP was excited with a filter cube containing a 490/10 nm excitation filter. A long-pass filter was used for emission measurements of light wavelengths $>424 \mathrm{~nm}$ (Fig. S2A). Spectral images were collected with exposure times ranging from 200 to $500 \mathrm{~ms}$ depending on the fluorescence intensity of the individual cell using the Evolve 512 EMCCD camera and MetaMorph software (Molecular Devices, Sunnyvale, CA). The exposure time was maintained the same for recording donor emission and acceptor emission spectra of the same FRET experiment. The spectral images were analyzed with ImageJ/Fiji (National Institutes of Health). The background fluorescence spectrum was subtracted using areas outside of the cell of interest. All microscopic experiments were done at room temperature.

FRET donor-only (Anap or CFP) constructs were used to obtain the averaged donor emission spectrum called $F$ (donor) excited by donor excitation wavelength. We then normalized the template emission of donor alone $F$ (donor) to the peak intensity of the $F_{C F P / Y F P}{ }^{\text {CFP }}$ or $F_{\text {Anap/YFP }}{ }^{\text {Anap }}$ spectrum, which is the emission spectrum upon the excitation using donor (in superscript) excitation wavelength when both donors and acceptors (in subscript) are present. The scaled $\mathrm{F}$ (donor) was then subtracted from either the $\mathrm{F}_{\mathrm{CFP} / \mathrm{YFP}}{ }^{\mathrm{CFP}}$ or the $\mathrm{F}_{\text {Anap/YFP }}{ }^{\text {Anap }}$ spectrum in each case, generating the spectrum $F_{F R E T}$. This subtraction was for correcting the bleed-through of FRET. Ratio $A$ was then calculated as Ratio $A=F_{F R E T} / F_{\text {Anap/YFP }}{ }^{\text {YFP }}$ for the Anap/YFP pair or Ratio $A=F_{F R E T} / F_{C F P / Y F P}{ }^{\text {YFP }}$ for the CFP/YFP pair. The denominator of the Ratio $A$ is the spectrum generated by using the excitation light at the YFP excitation wavelength. In addition, 
two emission spectra were collected from cells that were only transfected with YFP-containing constructs (in the absence of FRET donors): FYFP ${ }^{\text {CFP }}$ or FYFP ${ }^{A n a p}$, the emission spectrum using donor (CFP or Anap) excitation and FYFP YFP, the emission spectrum using acceptor (YFP)

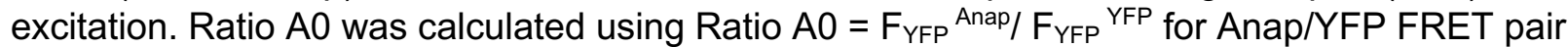
or Ratio $A 0=F_{Y F P}{ }^{C F P} / F_{Y F P}{ }^{Y F P}$ for CFP/YFP FRET pair. Ratio A0 corrects for the cross-talk of FRET, which is the fraction of the direct excitation of FRET acceptors by donor-excitation light. Absolute FRET efficiency is linearly proportional to the difference between Ratio A and Ratio A0, hence we call that apparent FRET efficiency = Ratio A - Ratio A0 $(49,68)$. Ratio A RatioAO $>0$ indicates a detectable FRET signal.

The relationship between the apparent FRET efficiency $(E)$ and the ratio $(R)$ of the FRET donor to acceptor peak intensity was fitted with a Langmuir isotherm (50): $E=E_{\max }{ }^{*}(R / R+K)$, where $\mathrm{E}_{\max }$ is the maximum apparent FRET efficiency, $\mathrm{K}$ is analogous to a dissociation constant for ligand binding (69).

\section{Statistics}

The results are presented as mean \pm SEM. Statistical significance was determined using the two-tailed Student's t test, ${ }^{*} p<0.05$.

\section{ACKNOWLEDGMENTS AND FUNDING SOURCES}

I thank Drs. Bertil Hille and William N. Zagotta for support, sharing equipment, and advice on the manuscript; Dr. Donald W. Hilgemann (University of Texas Southwestern) for helpful advice; Jongyun Myeong and members of the Hille lab and the Zagotta lab for discussions and suggestions. This work was supported by grants from the National Institutes of Health of the United States of America: R37NS08174 and R01EY010329. 


\section{FIGURE LEGENDS:}

\section{Figure 1. FRET between L-Anap incorporated in BACE1 and YFP-tagged KCNQ2/3} channels

(A) Sequence alignment of mouse, rat, and human BACE1 proteins highlighting the four cysteines for palmitoylation and the site of leucine $500\left({ }^{*}\right)$. The transmembrane region is in green. (B) Representative emission spectra used to calculate FRET between BACE1-L500Anap and KCNQ2/3-YFP. The cyan (donor only) and green spectra (both donor and acceptor) are generated by the Anap excitation wavelength; the yellow spectrum (with both donor and acceptor) by YFP excitation; the red spectrum is the subtraction of the cyan spectrum from the green spectrum (see methods). The ratio of the peak intensity of the red spectrum divided by that of the yellow spectrum (Ratio $A=F_{F R E T} / F_{\text {Anap/YFP }}{ }^{\text {YFP }}$ ) after subtracting the cross-talk (Ratio $\mathrm{A} 0=\mathrm{F}_{\mathrm{YFP}}{ }^{\text {Anap }} / \mathrm{F}_{\mathrm{YFP}}{ }^{\mathrm{YFP}}$ in panel $\mathrm{C}$ ) is proportional to the amount of FRET detected, therefore called apparent FRET efficiency = Ratio A - Ratio A0. (C) Representative emission spectrum of KCNQ2/3-YFP-only cells using direct YFP excitation (black, FYFP ${ }^{\text {YFP }}$ ) compared with the spectrum (blue, FYFP ${ }^{\text {Anap }}$ ) from the same sample using excitation for Anap. (D) Relationship between the apparent FRET efficiency and the ratios of the peak intensity (at around $475 \mathrm{~nm}$ ) of the green spectrum divided by that of the YFP spectrum (at $530 \mathrm{~nm}$ ) in panel B. (E) Summary of the apparent efficiencies for the FRET (mean of data with all the measured ratios of Anap/YFP in panel $D$ ) between $K C N Q 2 / 3$ and BACE1. It includes results with or without PANAP, BACE1$4 \mathrm{C} / \mathrm{A}$, in the presence of $0.1 \mathrm{mM} 2-\mathrm{BP}$ as well as FRET between KCNH channel and BACE1. Shown as mean \pm SEM, * $p<0.05$ independently compared to the "BACE1+KCNH" condition.

\section{Figure 2. FRET between Caveolin 1-CFP and KCNQ2/3-YFP channels}

(A-C) Representative emission spectra for calculating FRET between caveolin 1-CFP and KCNQ2/3-YFP. The cyan and green spectra are generated by illumination of the CFP excitation wavelength; the yellow spectrum by YFP excitation; the red spectrum is the subtraction of the cyan spectrum from the green spectrum. (D) Representative emission spectrum of KCNQ2/3YFP alone cells using direct YFP excitation versus the spectrum (smaller amplitude) from the same sample by the excitation for CFP. (E) Relationship between the apparent FRET efficiency (Ratio A- Ratio A0) and the ratio of peak intensities of CFP versus YFP for the FRET between KCNQ2/3 and caveolin 1 in the conditions with or without BACE1 or with BACE1-4C/A. (F) Summary of the apparent FRET efficiencies for the same conditions in panel E; abbreviation: CAV1, caveolin 1; data are shown as mean \pm SEM, ${ }^{*} p<0.05$.

Figure 3. Fluorescent probes that target to lipid rafts and non-rafts.

(A) Schematic illustration of L10, S15 as well as caveolin 1. Sites of myristoylation and palmitoylation are shown. The Poly-N sequence are the flexible linker connecting to a fluorescent protein (FP), CFP or YFP in this paper. (B) Representative emission spectra for the FRET between caveolin 1-CFP and L10-YFP. (C) Representative emission spectra for the FRET between caveolin 1-CFP and S15-YFP. (D) Relationship between the apparent FRET efficiency and the ratio of peak intensities of CFP versus YFP for the FRET pairs shown in panel $\mathrm{B}$ and $\mathrm{C}$.

Figure 4. Using L10, S15 to probe the localization of KCNQ2/3 channels. 
Relationship between the apparent FRET efficiency and the ratio CFP/YFP for the FRET between the L10-CFP (A) or S15-CFP (B) and the KCNQ2/3-YFP in the conditions with or without BACE1 or with BACE1-4C/A. (C) and (D) Summary of the apparent efficiencies for the conditions in panel $A$ and panel $B$, respectively (E) Relationship between the apparent FRET efficiency and the ratio CFP/YFP for the FRET between the L10-CFP and KCNQ2/3-YFP after treating cells with acutely-applied $5 \mathrm{mM} \mathrm{M \beta CD}, 1 \mathrm{mM}$ chloroform and $0.1 \mathrm{mM} 2-\mathrm{BP}$. The notreatment control condition, which is the same fitted curve as in the red curve in panel $A$ is shown for clarity. (F) Summary of the apparent FRET efficiencies for the same conditions in panel $E$; data are shown as mean \pm SEM, ${ }^{*} p<0.05$. (G) Cartoon illustration summarizing the probing of the localization of KCNQ2/3 channels using L10 and S15 marker.

Figure S1. Schematic illustration of L-Anap incorporation to BACE1

(A) Structure of L-Anap and the Amber stop-codon suppression strategy for incorporating LAnap. (B) Cartoon showing the FRET between BACE1-L500Anap and KCNQ2/3-YFP.

Figure S2. Procedure of performing spectral measurements of cultured cells

(A) Calibration of spectral measurements using different emission filters with Tungsten light illumination. (B) Flow-chart illustration of an example of measuring spectral FRET using CFPtagged and YFP-tagged constructs transfected to tsA cells.

\section{Figure S3. FRET between Tubby-YFP and L10-CFP or S15-CFP.}

(A) Representative emission spectra for the FRET between L10-CFP and Tubby-YFP, which labels $\mathrm{PI}(4,5) \mathrm{P}_{2}$. (B) Relationship between the apparent FRET efficiency and the ratio (CFP/YFP) for the FRET between the L10-CFP or S15-CFP with the Tubby-YFP. The same fitted curves shown in Figure $4 \mathrm{~A}$ and $\mathrm{B}$ for the FRET between L10-CFP or S15-CFP with the KCNQ2/3-YFP are included for comparison of FRET intensity. 


\section{REFERENCES}

1. Perl DP (2010) Neuropathology of Alzheimer's disease. Mt Sinai J Med 77(1):32-42.

2. Weller J \& Budson A (2018) Current understanding of Alzheimer's disease diagnosis and treatment. F1000Res 7.

3. Vassar R, et al. (2014) Function, therapeutic potential and cell biology of BACE proteases: current status and future prospects. J Neurochem 130(1):4-28.

4. Jonsson T, et al. (2012) A mutation in APP protects against Alzheimer's disease and age-related cognitive decline. Nature 488(7409):96-99.

5. Di Fede G, et al. (2009) A recessive mutation in the APP gene with dominant-negative effect on amyloidogenesis. Science 323(5920):1473-1477.

6. Vassar R \& Kandalepas PC (2011) The $\beta$-secretase enzyme BACE1 as a therapeutic target for Alzheimer's disease. Alzheimers Res Ther 3(3):20.

7. Cole SL \& Vassar R (2007) The Alzheimer's disease $\beta$-secretase enzyme, BACE1. Mol Neurodegener 2:22.

8. Kobayashi D, et al. (2008) BACE1 gene deletion: impact on behavioral function in a model of Alzheimer's disease. Neurobiol Aging 29(6):861-873.

9. Ou-Yang $\mathrm{MH}$, et al. (2018) Axonal organization defects in the hippocampus of adult conditional BACE1 knockout mice. Sci Transl Med 10(459).

10. Hu X, et al. (2006) BACE1 modulates myelination in the central and peripheral nervous system. Nat Neurosci 9(12):1520-1525.

11. Lombardo S, et al. (2019) BACE1 partial deletion induces synaptic plasticity deficit in adult mice. Sci Rep 9(1):19877.

12. Lehnert $\mathrm{S}$, et al. (2016) lon channel regulation by $\beta$-secretase BACE1-enzymatic and nonenzymatic effects beyond Alzheimer's disease. Channels (Austin) 10(5):365-378.

13. Kim DY, et al. (2007) BACE1 regulates voltage-gated sodium channels and neuronal activity. Nat Cell Biol 9(7):755-764.

14. Brown DA \& Adams PR (1980) Muscarinic suppression of a novel voltage-sensitive $\mathrm{K}^{+}$current in a vertebrate neurone. Nature 283(5748):673-676.

15. Hessler S, et al. (2015) $\beta$-Secretase BACE1 regulates hippocampal and reconstituted M-currents in a $\beta$-subunit-like fashion. J Neurosci 35(8):3298-3311.

16. Wang HS, et al. (1998) KCNQ2 and KCNQ3 potassium channel subunits: molecular correlates of the M-channel. Science 282(5395):1890-1893.

17. Abbott GW (2020) KCNQs: Ligand- and Voltage-Gated Potassium Channels. Front Physiol 11:583.

18. Hernandez CC, Zaika O, Tolstykh GP, \& Shapiro MS (2008) Regulation of neural KCNQ channels: signalling pathways, structural motifs and functional implications. J Physiol 586(7):1811-1821.

19. Shah M, Mistry M, Marsh SJ, Brown DA, \& Delmas P (2002) Molecular correlates of the Mcurrent in cultured rat hippocampal neurons. J Physiol 544(Pt 1):29-37.

20. Yue C \& Yaari Y (2004) KCNO/M channels control spike afterdepolarization and burst generation in hippocampal neurons. J Neurosci 24(19):4614-4624.

21. Klinger F, Gould G, Boehm S, \& Shapiro MS (2011) Distribution of M-channel subunits KCNQ2 and KCNQ3 in rat hippocampus. Neuroimage 58(3):761-769.

22. Carver CM, Hastings SD, Cook ME, \& Shapiro MS (2020) Functional responses of the hippocampus to hyperexcitability depend on directed, neuron-specific KCNQ2 $\mathrm{K}^{+}$channel plasticity. Hippocampus 30(5):435-455.

23. Biervert C, et al. (1998) A potassium channel mutation in neonatal human epilepsy. Science 279(5349):403-406. 
24. Singh NA, et al. (1998) A novel potassium channel gene, KCNQ2, is mutated in an inherited epilepsy of newborns. Nat Genet 18(1):25-29.

25. Vossel KA, Tartaglia MC, Nygaard HB, Zeman AZ, \& Miller BL (2017) Epileptic activity in Alzheimer's disease: causes and clinical relevance. Lancet Neurol 16(4):311-322.

26. Cordy JM, Hussain I, Dingwall C, Hooper NM, \& Turner AJ (2003) Exclusively targeting $\beta$ secretase to lipid rafts by $\mathrm{GPI}$-anchor addition up-regulates $\beta$-site processing of the amyloid precursor protein. Proc Natl Acad Sci U S A 100(20):11735-11740.

27. Ehehalt R, Keller P, Haass C, Thiele C, \& Simons K (2003) Amyloidogenic processing of the Alzheimer $\beta$-amyloid precursor protein depends on lipid rafts. J Cell Biol 160(1):113-123.

28. Simons K \& Ikonen E (1997) Functional rafts in cell membranes. Nature 387(6633):569-572.

29. Lingwood D \& Simons K (2010) Lipid rafts as a membrane-organizing principle. Science 327(5961):46-50.

30. Kojro E, Gimpl G, Lammich S, Marz W, \& Fahrenholz F (2001) Low cholesterol stimulates the nonamyloidogenic pathway by its effect on the $\alpha$-secretase ADAM 10. Proc Natl Acad Sci U S A 98(10):5815-5820.

31. Cordy JM, Hooper NM, \& Turner AJ (2006) The involvement of lipid rafts in Alzheimer's disease. Mol Membr Biol 23(1):111-122.

32. Linder ME \& Deschenes RJ (2007) Palmitoylation: policing protein stability and traffic. Nat Rev Mol Cell Biol 8(1):74-84.

33. Vetrivel KS, et al. (2009) Alzheimer disease $A \beta$ production in the absence of S-palmitoylationdependent targeting of BACE1 to lipid rafts. J Biol Chem 284(6):3793-3803.

34. Chung HJ, Jan YN, \& Jan LY (2006) Polarized axonal surface expression of neuronal KCNQ channels is mediated by multiple signals in the KCNQ2 and KCNQ3 C-terminal domains. Proc Natl Acad Sci U S A 103(23):8870-8875.

35. Zhang J \& Shapiro MS (2016) Mechanisms and dynamics of AKAP79/150-orchestrated multiprotein signalling complexes in brain and peripheral nerve. J Physiol 594(1):31-37.

36. He M, Jenkins $P, \&$ Bennett V (2012) Cysteine 70 of ankyrin-G is S-palmitoylated and is required for function of ankyrin-G in membrane domain assembly. J Biol Chem 287(52):43995-44005.

37. Keith DJ, et al. (2012) Palmitoylation of A-kinase anchoring protein 79/150 regulates dendritic endosomal targeting and synaptic plasticity mechanisms. J Neurosci 32(21):7119-7136.

38. Buggia-Prevot $V$, et al. (2014) Axonal BACE1 dynamics and targeting in hippocampal neurons: a role for Rab11 GTPase. Mol Neurodegener 9:1.

39. Rodgers W (2002) Making membranes green: construction and characterization of GFP-fusion proteins targeted to discrete plasma membrane domains. Biotechniques 32(5):1044-1046, 1048, 1050-1041.

40. Chichili GR \& Rodgers W (2007) Clustering of membrane raft proteins by the actin cytoskeleton. J Biol Chem 282(50):36682-36691.

41. Chatterjee A, Guo J, Lee HS, \& Schultz PG (2013) A genetically encoded fluorescent probe in mammalian cells. J Am Chem Soc 135(34):12540-12543.

42. Kalstrup $T$ \& Blunck $R$ (2013) Dynamics of internal pore opening in $\mathrm{K}_{\mathrm{v}}$ channels probed by a fluorescent unnatural amino acid. Proc Natl Acad Sci U S A 110(20):8272-8277.

43. Andrew RJ, et al. (2017) Lack of BACE1 S-palmitoylation reduces amyloid burden and mitigates memory deficits in transgenic mouse models of Alzheimer's disease. Proc Natl Acad Sci U S A 114(45):E9665-E9674.

44. Toh WH, Chia PZC, Hossain MI, \& Gleeson PA (2018) GGA1 regulates signal-dependent sorting of BACE1 to recycling endosomes, which moderates A $\beta$ production. Mol Biol Cell 29(2):191-208. 
45. Pastorino L, Ikin AF, Nairn AC, Pursnani A, \& Buxbaum JD (2002) The carboxyl-terminus of BACE contains a sorting signal that regulates BACE trafficking but not the formation of total $A \beta$. Mol Cell Neurosci 19(2):175-185.

46. Gordon SE, Munari M, \& Zagotta WN (2018) Visualizing conformational dynamics of proteins in solution and at the cell membrane. Elife 7.

47. Dai G, Aman TK, DiMaio F, \& Zagotta WN (2019) The HCN channel voltage sensor undergoes a large downward motion during hyperpolarization. Nat Struct Mol Biol.

48. Dai G \& Zagotta WN (2017) Molecular mechanism of voltage-dependent potentiation of KCNH potassium channels. Elife 6 .

49. Takanishi CL, Bykova EA, Cheng W, \& Zheng J (2006) GFP-based FRET analysis in live cells. Brain Res 1091(1):132-139.

50. Zacharias DA, Violin JD, Newton AC, \& Tsien RY (2002) Partitioning of lipid-modified monomeric GFPs into membrane microdomains of live cells. Science 296(5569):913-916.

51. Bykova EA, Zhang XD, Chen TY, \& Zheng J (2006) Large movement in the C terminus of CLC-0 chloride channel during slow gating. Nat Struct Mol Biol 13(12):1115-1119.

52. Cheng W, Yang F, Takanishi CL, \& Zheng J (2007) Thermosensitive TRPV channel subunits coassemble into heteromeric channels with intermediate conductance and gating properties. $J$ Gen Physiol 129(3):191-207.

53. Davda D, et al. (2013) Profiling targets of the irreversible palmitoylation inhibitor 2bromopalmitate. ACS Chem Biol 8(9):1912-1917.

54. Li S, Song KS, \& Lisanti MP (1996) Expression and characterization of recombinant caveolin. Purification by polyhistidine tagging and cholesterol-dependent incorporation into defined lipid membranes. J Biol Chem 271(1):568-573.

55. Williams TM \& Lisanti MP (2004) The caveolin proteins. Genome Biol 5(3):214.

56. Mahammad S \& Parmryd I (2015) Cholesterol depletion using methyl- $\beta$-cyclodextrin. Methods Mol Biol 1232:91-102.

57. Pavel MA, Petersen EN, Wang H, Lerner RA, \& Hansen SB (2020) Studies on the mechanism of general anesthesia. Proc Natl Acad Sci U S A 117(24):13757-13766.

58. Suh $B C \&$ Hille B (2008) PIP $P_{2}$ is a necessary cofactor for ion channel function: how and why? Annu Rev Biophys 37:175-195.

59. Suh BC \& Hille B (2002) Recovery from muscarinic modulation of M current channels requires phosphatidylinositol 4,5-bisphosphate synthesis. Neuron 35(3):507-520.

60. Dai G, Yu H, Kruse M, Traynor-Kaplan A, \& Hille B (2016) Osmoregulatory inositol transporter SMIT1 modulates electrical activity by adjusting PI(4,5) $\mathrm{P}_{2}$ levels. Proc Natl Acad Sci U S A 113(23):E3290-3299.

61. McCarthy AE, Yoshioka C, \& Mansoor SE (2019) Full-Length P2X ${ }_{7}$ Structures Reveal How Palmitoylation Prevents Channel Desensitization. Cell 179(3):659-670 e613.

62. Howie J, et al. (2014) Substrate recognition by the cell surface palmitoyl transferase DHHC5. Proc Natl Acad Sci U S A 111(49):17534-17539.

63. Hilgemann DW, et al. (2018) Lipid signaling to membrane proteins: From second messengers to membrane domains and adapter-free endocytosis. J Gen Physiol 150(2):211-224.

64. Gamper N, et al. (2006) Oxidative modification of M-type $\mathrm{K}^{+}$channels as a mechanism of cytoprotective neuronal silencing. EMBO J 25(20):4996-5004.

65. Lin MJ, et al. (2013) Massive palmitoylation-dependent endocytosis during reoxygenation of anoxic cardiac muscle. Elife 2:e01295.

66. Gu N, Vervaeke K, Hu H, \& Storm JF (2005) Kv7/KCNO/M and HCN/h, but not K 2 2/SK channels, contribute to the somatic medium after-hyperpolarization and excitability control in CA1 hippocampal pyramidal cells. J Physiol 566(Pt 3):689-715. 
bioRxiv preprint doi: https://doi.org/10.1101/2020.09.30.321307; this version posted January 4, 2021. The copyright holder for this preprint (which was not certified by peer review) is the author/funder. All rights reserved. No reuse allowed without permission.

67. Tzingounis AV \& Nicoll RA (2008) Contribution of KCNQ2 and KCNQ3 to the medium and slow afterhyperpolarization currents. Proc Natl Acad Sci U S A 105(50):19974-19979.

68. Erickson MG, Alseikhan BA, Peterson BZ, \& Yue DT (2001) Preassociation of calmodulin with voltage-gated $\mathrm{Ca}^{2+}$ channels revealed by FRET in single living cells. Neuron 31(6):973-985.

69. Kenworthy AK \& Edidin M (1998) Distribution of a glycosylphosphatidylinositol-anchored protein at the apical surface of MDCK cells examined at a resolution of $<100 \AA$ using imaging fluorescence resonance energy transfer. J Cell Biol 142(1):69-84. 
Figure 1

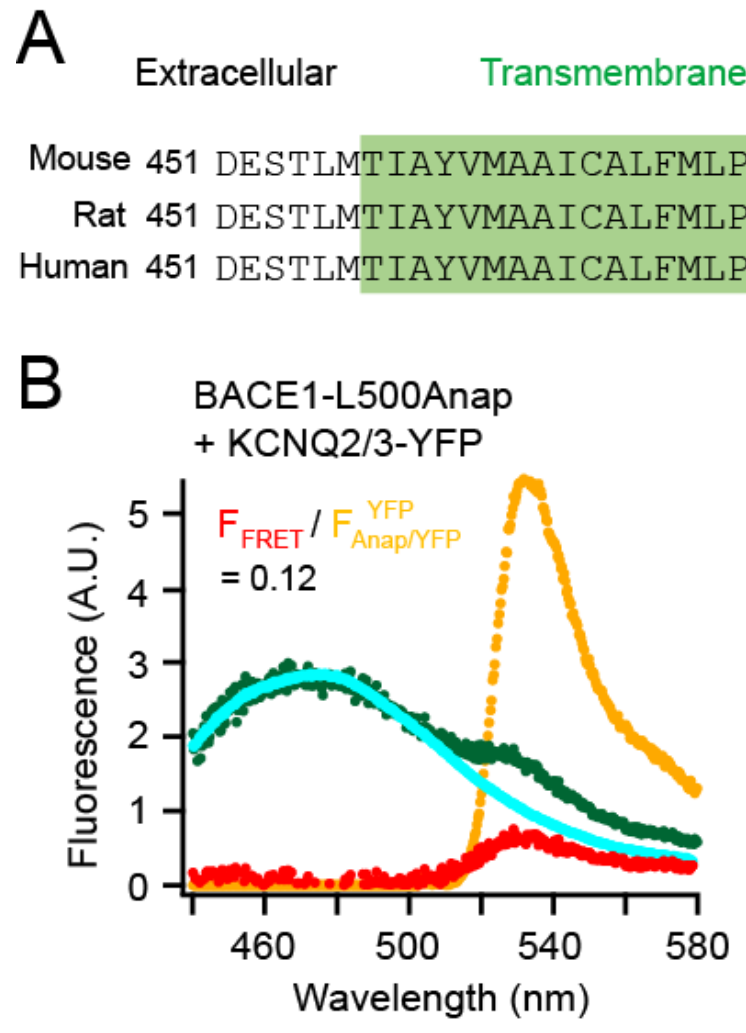

$$
474 \quad 478 \quad 482485
$$

Cytoplasmic

*

R Human 451 DESTLMTIAYVMAAICALFMLPLCLMVCQWRCLRCLRQQHDDFADDISLLK

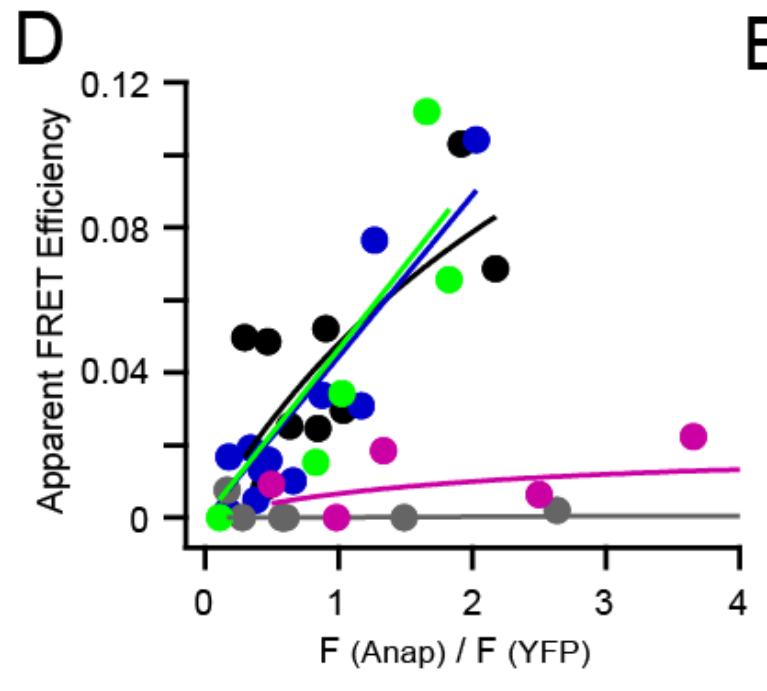

C KCNQ2/3-YFP alone
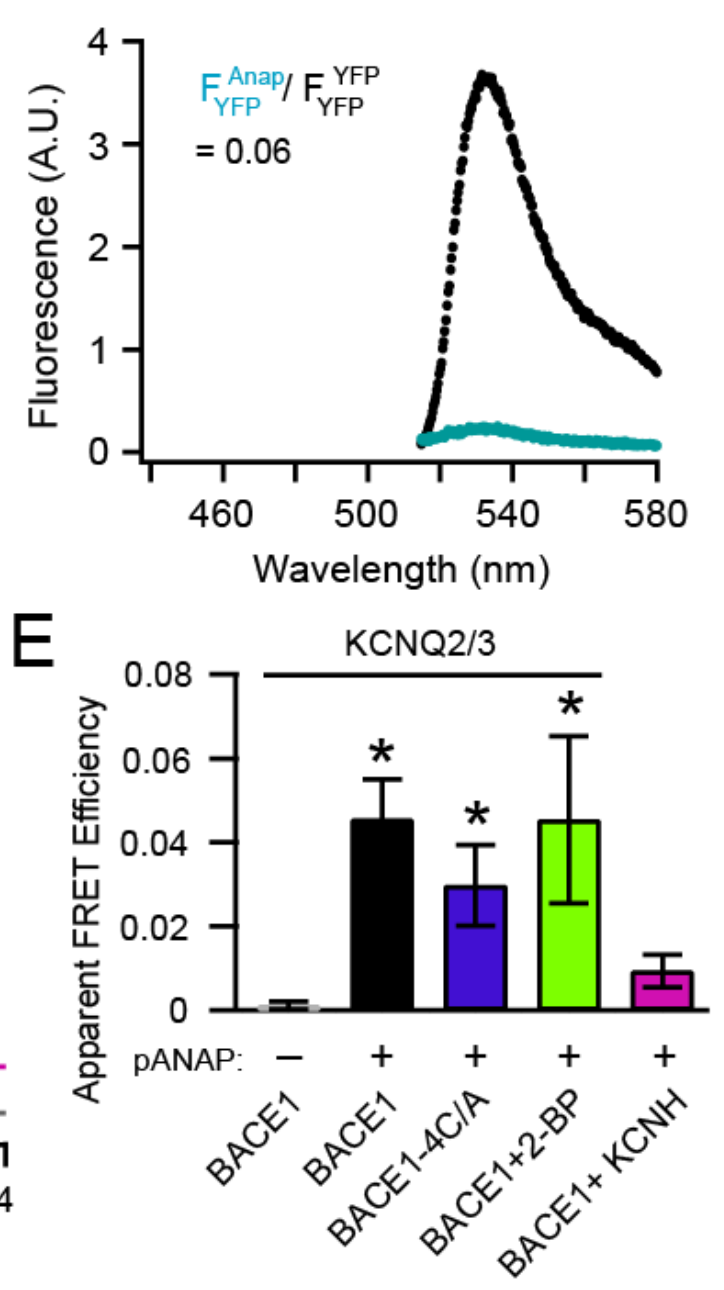
Figure 2
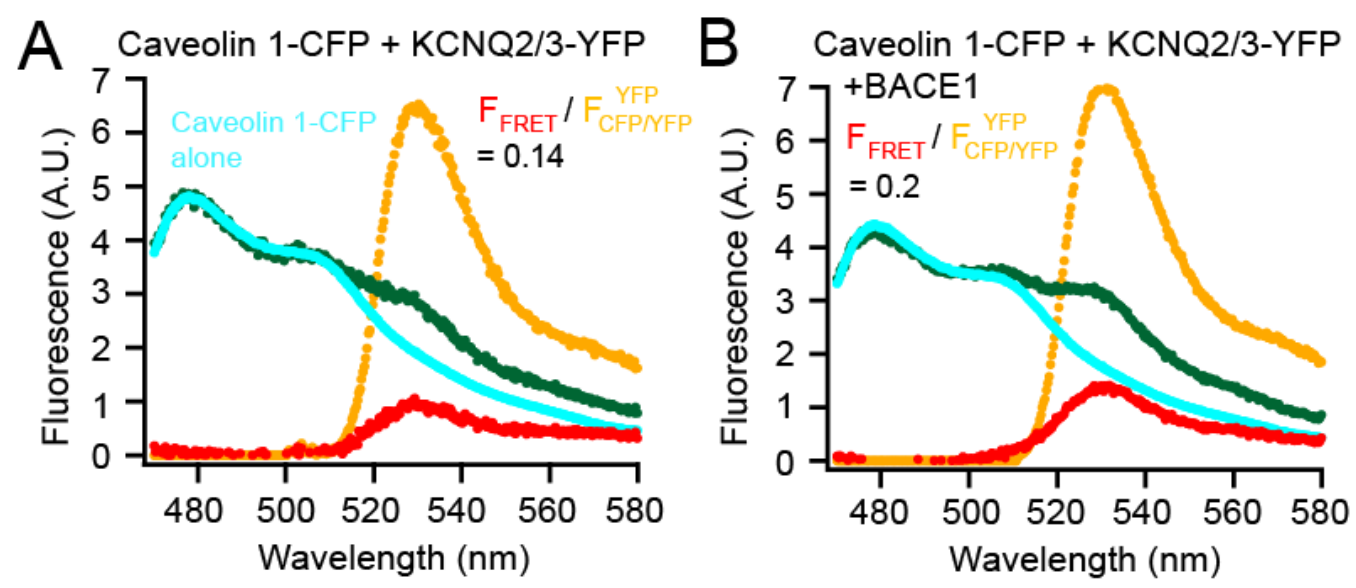

C Caveolin 1-CFP + KCNQ2/3-YFP
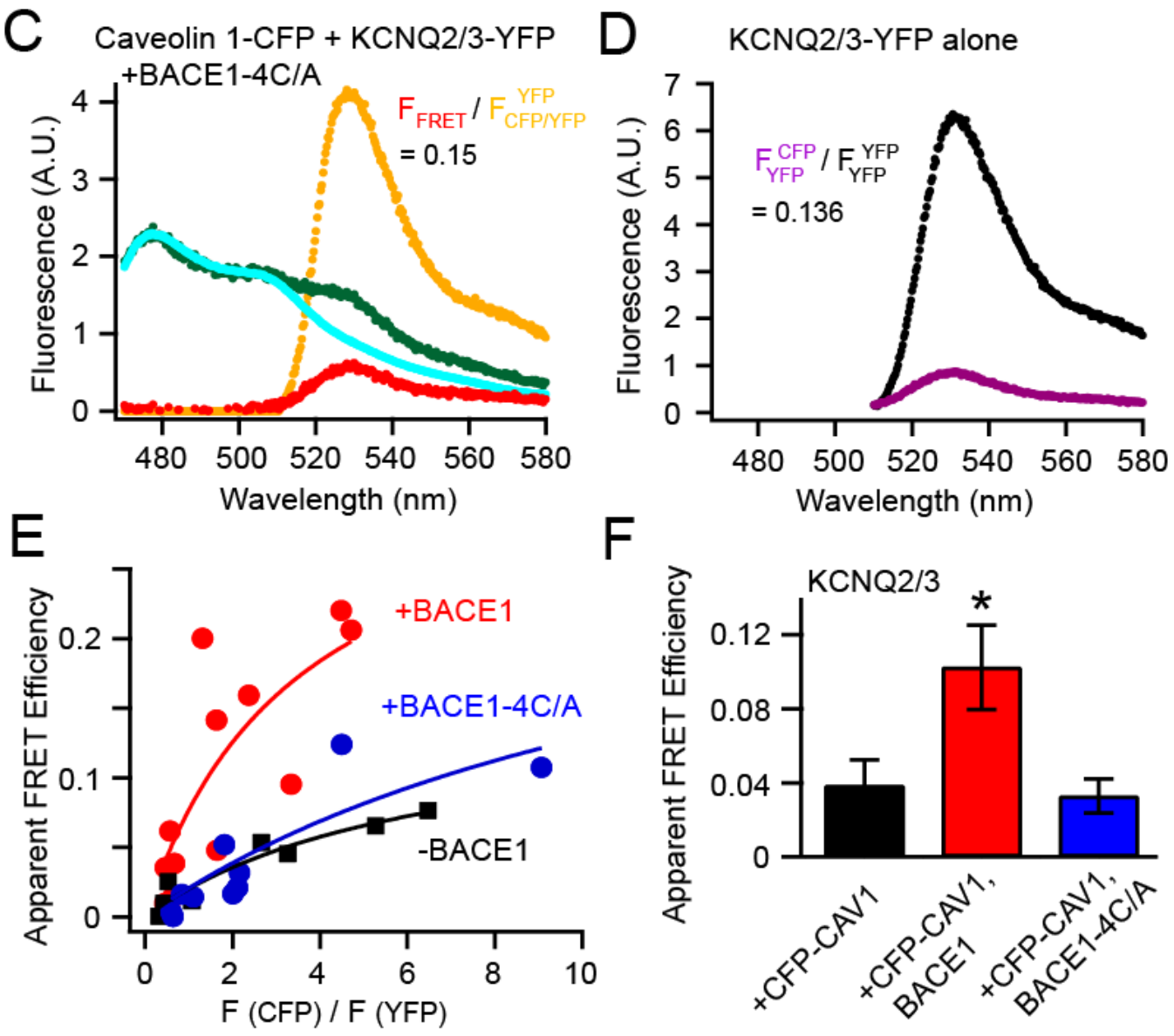

$\mathrm{F}$

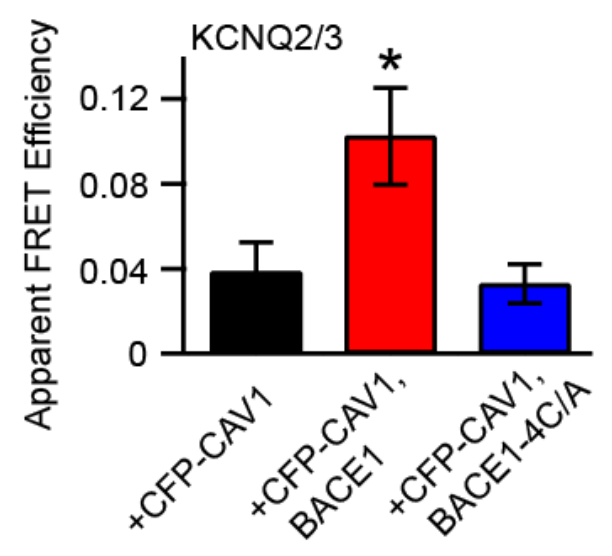


Figure 3

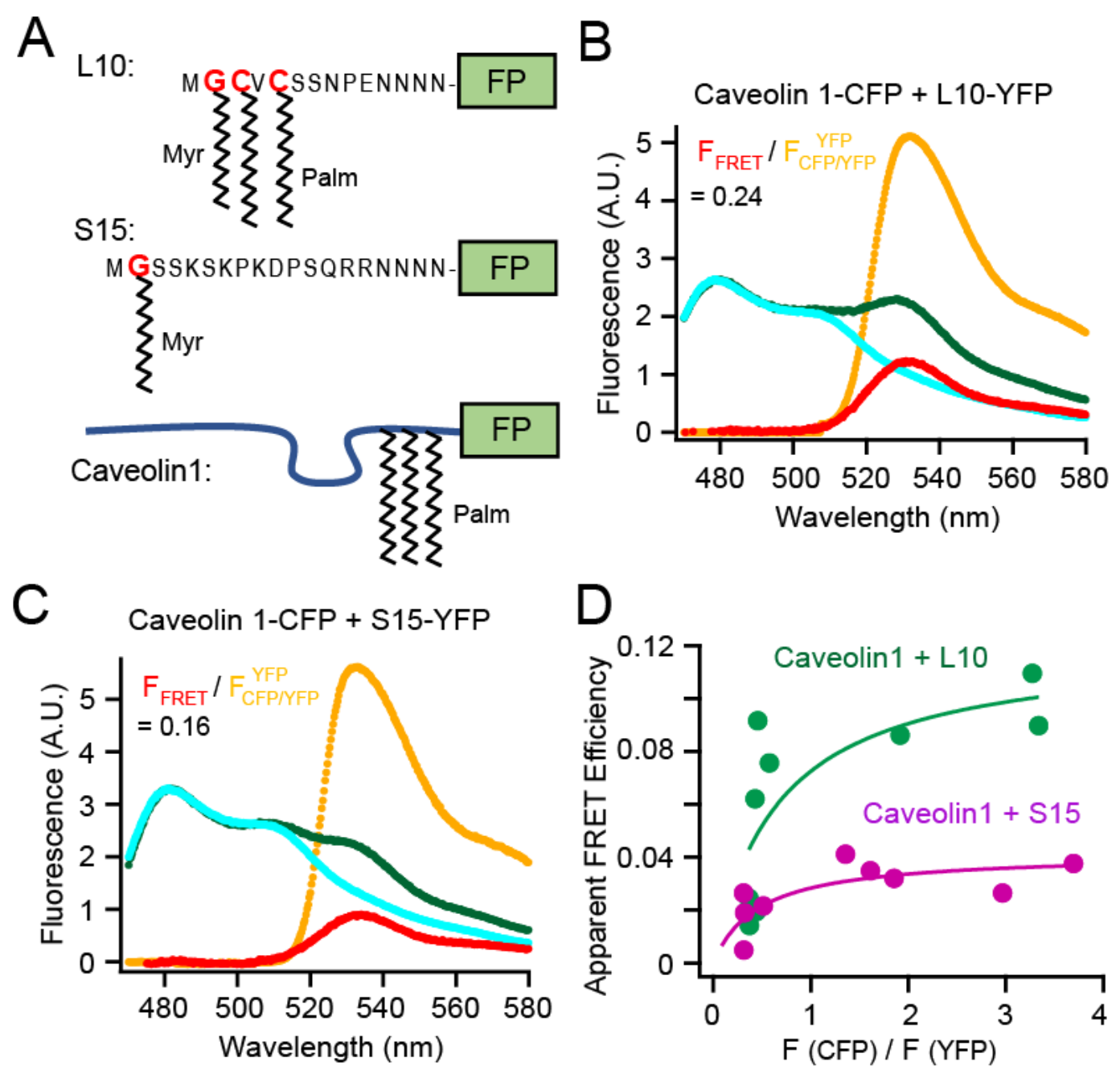


Figure 4
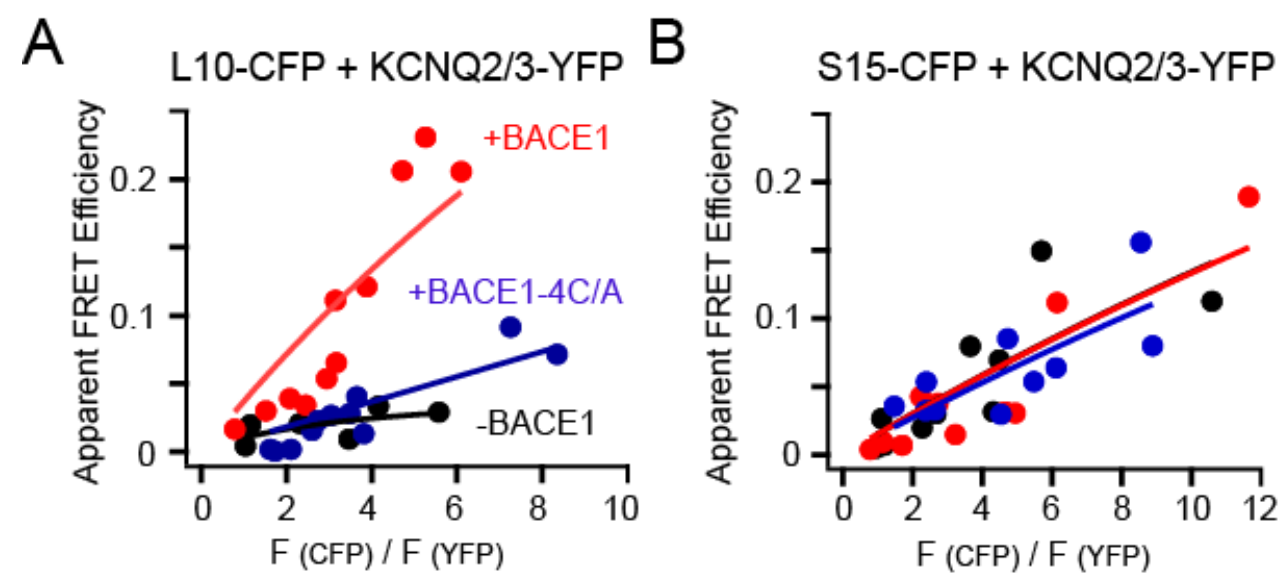

C

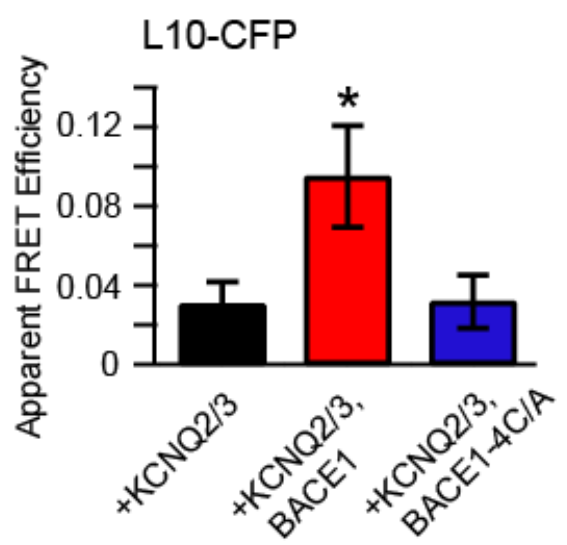

D

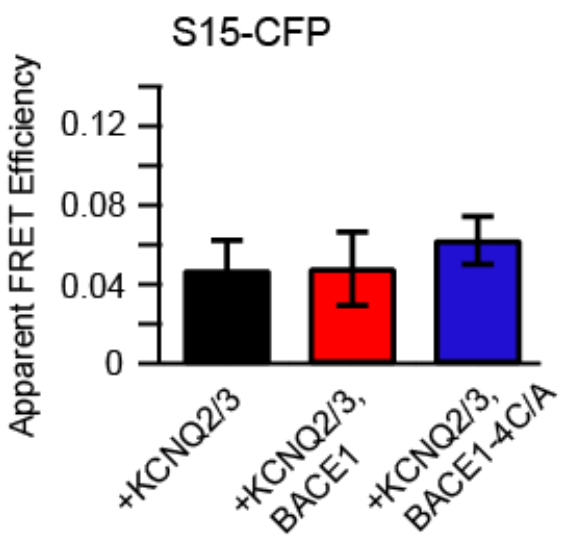

E

F
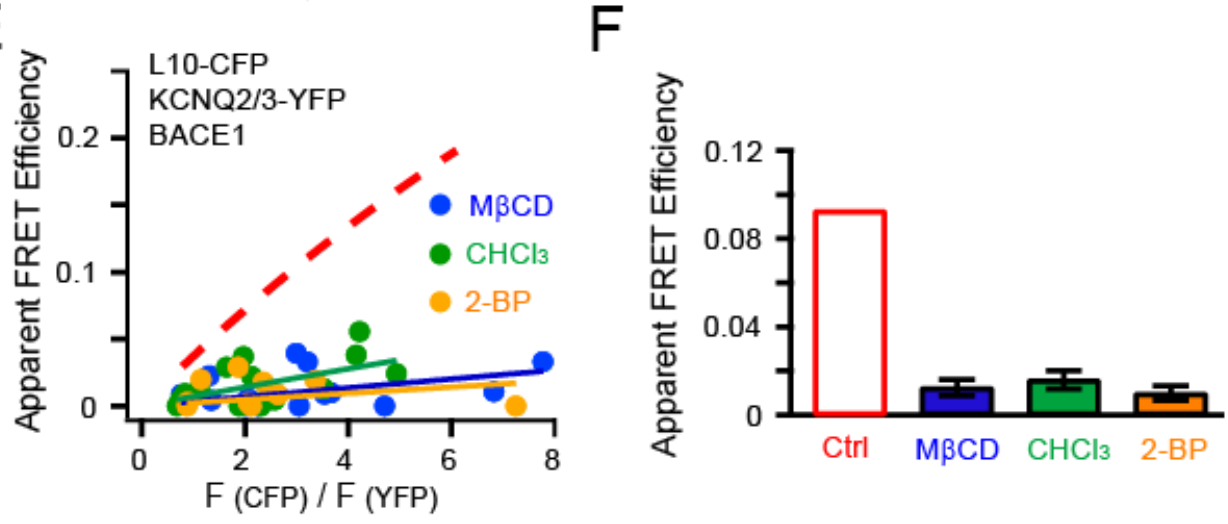

$G$
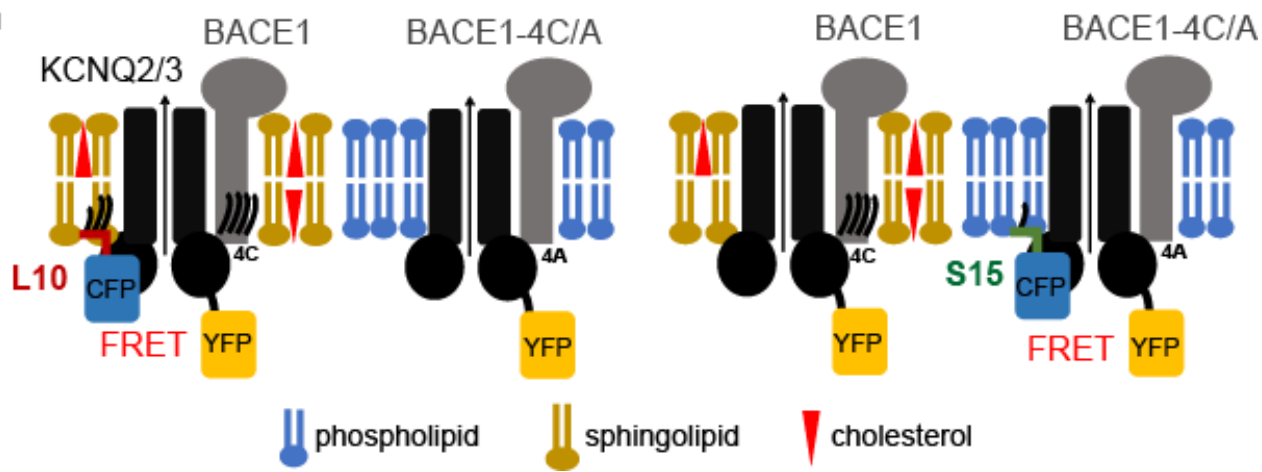
Figure $\mathbf{S 1}$
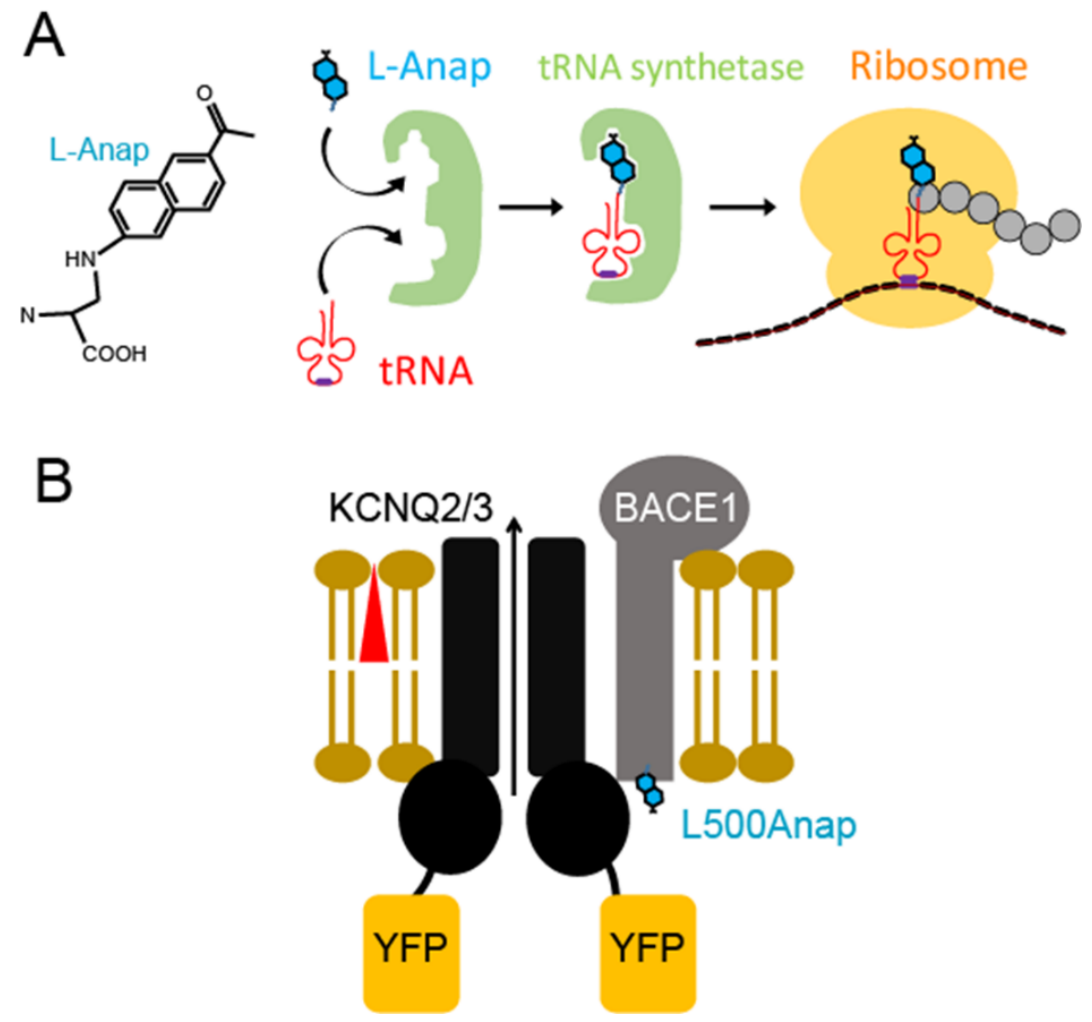


\section{Figure S2}

A

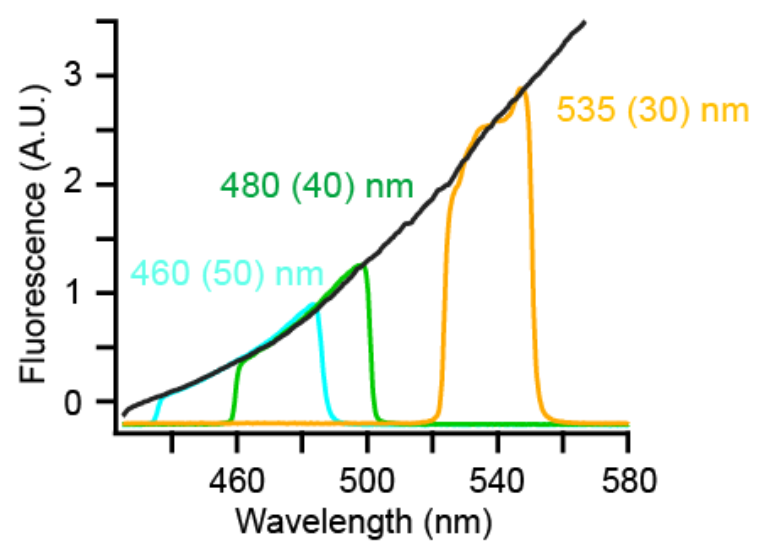

B

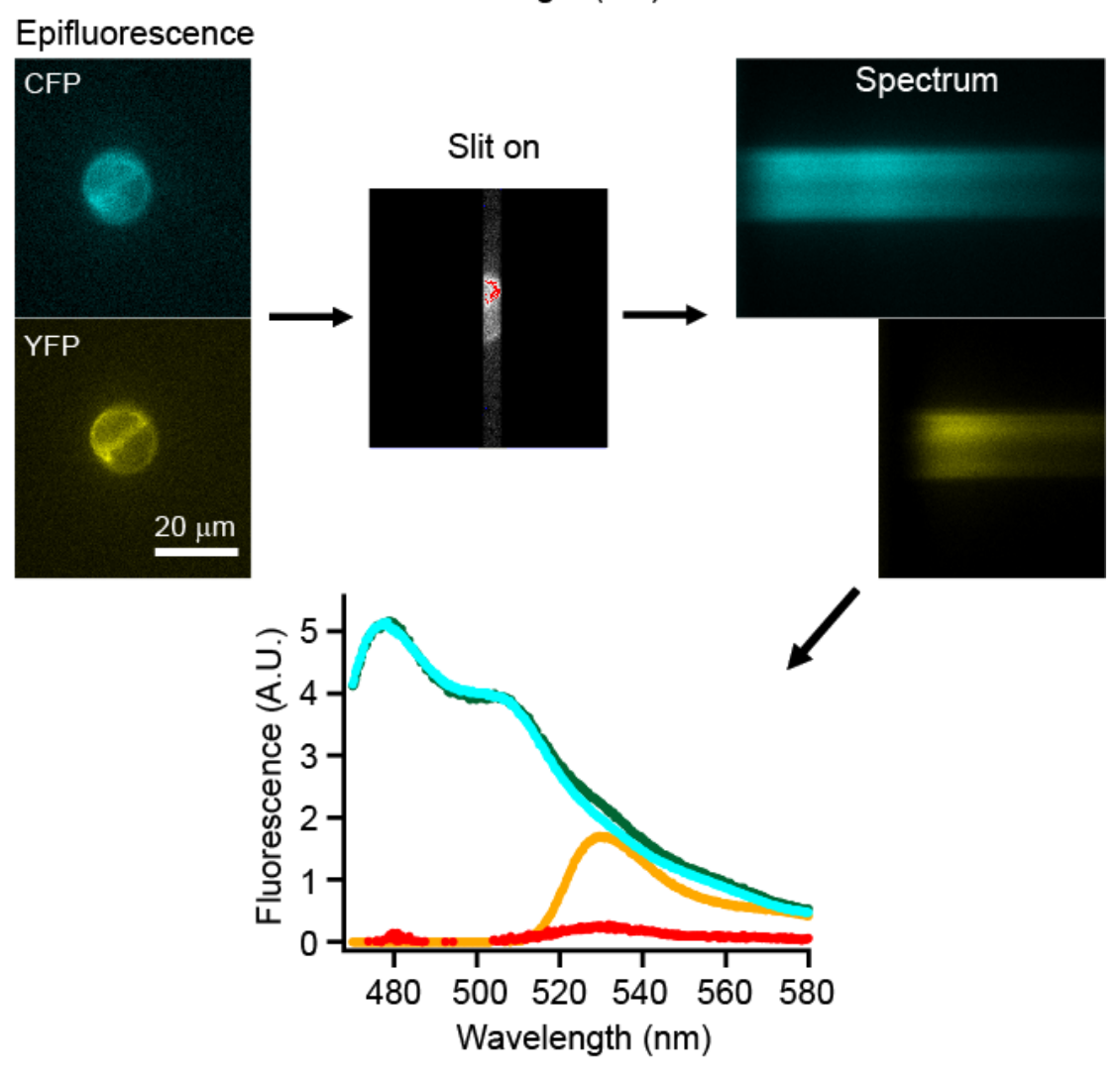


Figure S3
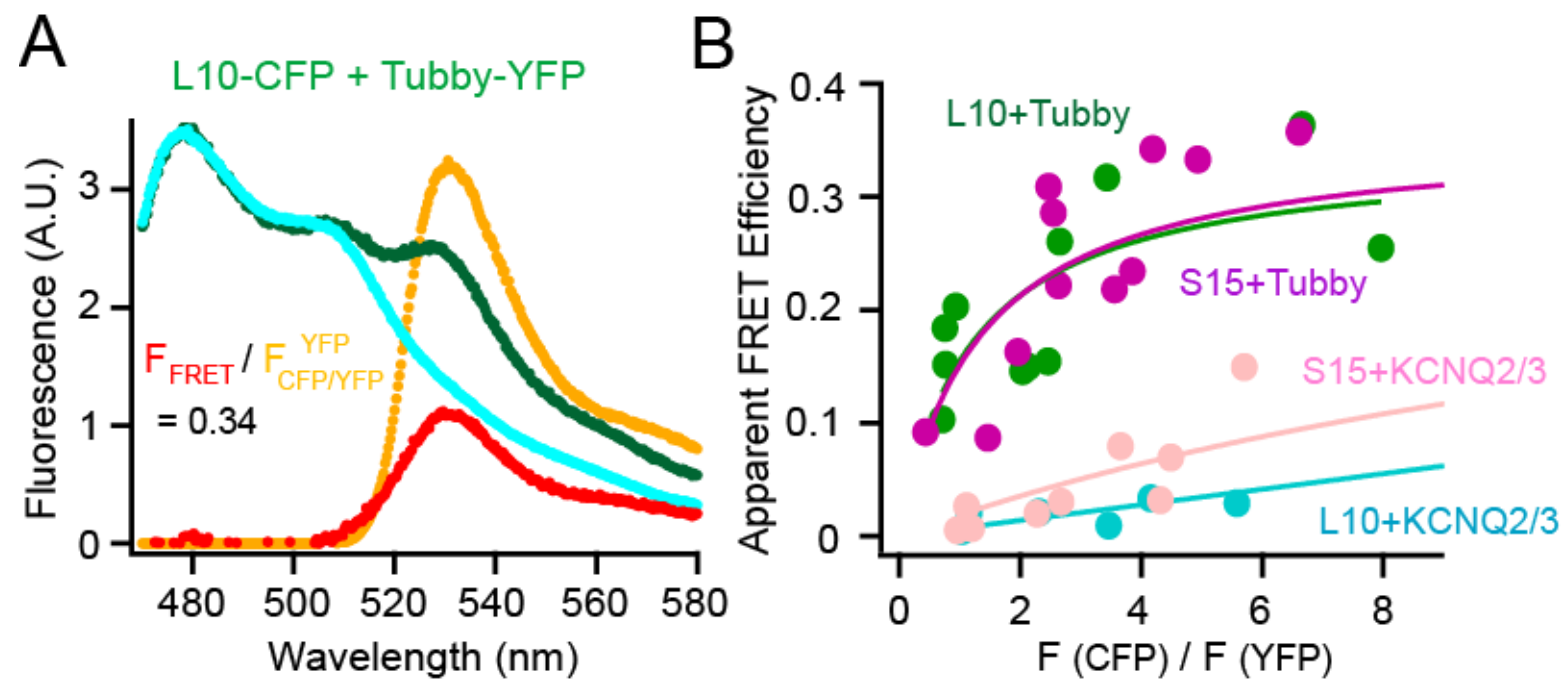